\title{
The relative importance of conidia and ascospores as primary inoculum of Venturia inaequalis in a Southeast England orchard
}

Article

Accepted Version

Passey, T. A. J., Robinson, D. J., Shaw, M. and Xu, X.-M. (2017) The relative importance of conidia and ascospores as primary inoculum of Venturia inaequalis in a Southeast England orchard. Plant Pathology, 66 (9). pp. 1445-1451. ISSN 0032-0862 doi: https://doi.org/10.1111/ppa.12686 Available at https://centaur.reading.ac.uk/69173/

It is advisable to refer to the publisher's version if you intend to cite from the work. See Guidance on citing.

To link to this article DOI: http://dx.doi.org/10.1111/ppa.12686

Publisher: Wiley-Blackwell

All outputs in CentAUR are protected by Intellectual Property Rights law, including copyright law. Copyright and IPR is retained by the creators or other copyright holders. Terms and conditions for use of this material are defined in the End User Agreement. 


\section{CentAUR}

Central Archive at the University of Reading

Reading's research outputs online 
1 Title: The relative importance of conidia and ascospores as primary 2 inoculum of Venturia inaequalis in a Southeast England orchard

3

4 Authors: T. A. J. Passey ${ }^{a b} *$, J. D. Robinson ${ }^{a}$, M. W. Shaw ${ }^{b}$, X.-M. Xu ${ }^{a}$

$5{ }^{a}$ NIAB EMR, New Road, East Malling, Kent, ME19 6BJ, UK; ${ }^{b}$ School of Agriculture, Policy

6 and Development, University of Reading, Reading, RG6 6AR, UK

$7 \quad *$ E-mail: tom.passey@emr.ac.uk

8

9 Running head: Apple scab primary inoculum

10

11 Key Words: Apple scab, Spilocaea pomi, Fusicladium dendriticum, asexual over-wintering 


\section{Abstract}

Apple scab, caused by Venturia inaequalis, can lead to large losses of marketable fruit if left uncontrolled. The disease appears in orchards during spring as lesions on leaves. These primary lesions are caused by spores released at bud burst from over-wintering sources; these spores can be sexually produced ascospores from the leaf litter or asexual conidia from mycelium in wood scab or within buds. We investigated the relative importance of conidia and ascospores as primary inoculum in an orchard in the United Kingdom. Potted trees not previously exposed to apple scab were placed next to $(\mathrm{c} .1 \mathrm{~m})$ orchard trees to trap airdispersed ascospores. Number and position of scab lesions were assessed on shoots from both the potted trees (infection by airborne ascospores) and neighbouring orchard trees (infection by both ascospores and splash-dispersed conidia overwintered in buds). The distribution and population similarity of scab lesions were compared in the two tree types by molecular analysis and through modelling of scab incidence and count data. Molecular analysis was inconclusive. Statistical modelling of results suggested that conidia may have contributed approximately $20-50 \%$ of the total primary inoculum in this orchard: incidence was estimated to be reduced by $20 \%$ on potted trees, and lesion number by $50 \%$. These results indicate that, although conidia are still a minority contributor to primary inoculum, their contribution in this orchard is sufficient to review current management. This might also be true of orchards with a similar climate.

\section{Introduction}

Annual epidemics of apple scab, caused by the ascomycete Venturia inaequalis, lead to large losses of marketable fruit worldwide if uncontrolled. The $V$. inaequalis life cycle sees overwintered spores released in the spring to infect newly emerged leaves. Lesions from these infections produce conidia which are dispersed by water splash, leading to secondary 
37 infections which in turn continue the secondary inoculum cycle throughout the growing season (MacHardy, 1996). There are two possible sources of over-wintered inoculum, one sexual and the other asexual. Ascospores, released during spring rainfall from leaf litter and wind dispersed, have traditionally been believed to be the most important primary inoculum of $V$. inaequalis. As a result the majority of research into apple scab control has focused on reducing leaf litter in orchards (Sutton et al., 2000; Vincent et al., 2004; Gomez et al., 2007) and inoculum forecasting based on ascospore development and release to aid the application of chemical control (Gadoury \& MacHardy, 1986; Beresford \& Manktelow, 1994; Berrie \& $\mathrm{Xu}, 2003)$.

$V$. inaequalis can also overwinter as stromata on twigs or as viable inoculum (most likely conidia) between bud scales (Cook, 1974; Hill, 1975; Becker et al., 1992).. It is likely asexual conidia are either washed on to leaves near to the source of overwintered scab, or they germinate and form a lesion on or around the bud forming conidia that are then released and dispersed by water. As with conidia from lesions in the main epidemic phase, these conidia from over-wintered sources will infect within an area close to the initial lesion site. In contrast, airborne ascospores will be turbulently dispersed or advected over longer distances . Thus, we would expect more leaves to be infected within the same flower truss or extension shoot, and more aggregation in lesions on individual leaves, if conidia are the primary inoculum. Studies (Holb et al., 2004, 2005; Gao et al., 2009) suggest that these conidial sources may be a significant part of the primary inoculum. This is important because reduction of overwintering inoculum and early season control measures differ for the two sources and because relatively lower levels of sexual reproduction (compared to all primary infections resulting from ascospores) in the population may affect the evolution of for pathogen virulence and fungicide resistance. 
If conidia contribute to primary inoculum it means that a proportion of the lesions present in an orchard are not recombinant products of meiosis. As a result the population as a whole will evolve at a different rate from the population of an orchard where ascospores are the sole primary inoculum since a certain proportion of the primary inoculum has identical genotypes to the previous year. The size of this change will depend on the genetic architecture of the trait under study. Furthermore, if a race of scab with superior fitness caused by several weakly linked polymorphic loci develops in an orchard it is likely to become dominant in the orchard faster, as more of the primary inoculum in successive seasons will be the favoured genotype. Fitness might be increased because of virulence towards resistant cultivars or resistance to a fungicide -

We aimed to investigate the relative importance of conidia and ascospores as sources of primary inoculum in an orchard in Southeast England. As previously stated, conidia are dispersed by water splash, but it is difficult to trap conidia from buds reliably in splash water in the early season. Instead, we placed potted trees in an orchard with a history of scab. Scab on potted trees not previously exposed to scab should result from ascospores because they are air-borne and travel longer distances, whereas scab on orchard trees may result from both ascospores and overwintered conidia. We compared scab incidence and clustering on the two types of recipient tree. We inferred the relative importance of the two sources of initial infection under the assumptions that young leaves from both types of trees are equally susceptible, and that conidia and ascospores have an equal infection potential/efficiency. The latter assumption is realistic for temperatures in the spring in the Southeast England and the infection requirement for conidia and scab (MacHardy, 1996). In addition, we compared the genetic structure of the $V$. inaequalis populations from potted and orchard trees using simple sequence repeat (SSR) markers. 


\section{Sampling and lesion assessments}

Orchard WM132 at East Malling Research (Kent, UK) has three consecutive rows of Malus x domestica 'Cox's Orange Pippin' (Cox) next to 3 consecutive rows of Malus $\mathrm{x}$ domestica 'Royal Gala' (Gala) on M9 rootstocks (rows $4 \mathrm{~m}$ apart); each row has 12 trees planted $1.75 \mathrm{~m}$ apart. This orchard is not sprayed with fungicides, but is pruned, and is c. 15 years old. Six potted trees of each of Cox and Gala on M9 rootstocks (c. 10-12 years old) in 10 litre pots were placed within the orchard trees of the same cultivar, two positions randomly chosen in each row, at bud burst in 2012, 2013 and 2014 (these positions remained the same for all three years); therefore observations were carried out between paired samples, a potted tree with a partner orchard tree. Potted trees had been kept in a polytunnel, except for the experimental exposure period, to prevent surface wetness and so prevent $V$. inaequalis infection (hence remove the possibility of overwintering conidia from previous years). The distance between the potted tree and the nearest orchard tree was c. $1 \mathrm{~m}$; potted trees were secured to the post of an orchard tree but the trees were arranged and pruned so that no branches of a potted tree touched or were directly above a branch of the corresponding orchard tree. Trees of both types were around 180-200 cm tall, with lowest shoots about $80 \mathrm{~cm}$ above ground level. Potted trees were watered (approx. 500ml) three times a week, directly onto the compost in the pot. The potted trees were returned to a polytunnel after sufficient infection events (3 to 5 weeks depending on weather), but before the first generation of conidia (i.e. visible lesions resulting from infection by primary inoculum) was produced, to ensure that infection on the potted trees all resulted from primary sources. The number of potential infection periods were 12, 3 and 3 for 2012, 2013 and 2014, respectively. Two weeks later, up to 15 shoots (flower trusses) were randomly sampled from across each potted tree and the nearest orchard tree (all available shoots were sampled when less than 15 
were available). The number of scab lesions was counted on both sides of every leaf and the position of infected leaves on the shoot noted. On the few occasions when the scab was severe enough that discrete lesions could not be defined an estimate of the percentage of leaf covered in scab was made and this converted to an estimated number of lesions (assuming a single lesion corresponds to $1 \%$ scabbed area, based on empirical experiences). From each infected leaf the most clearly separated scab lesion was selected and cut out with a $5 \mathrm{~mm}$ cork-borer, placed in a $2 \mathrm{ml}$ micro tube, left to air dry at room temperature and then transferred to a $-20^{\circ} \mathrm{C}$ freezer until DNA extraction.

\section{DNA extraction and screening}

DNA was extracted from six lesions (where possible) per tree, no more than one lesion from any one shoot. As lesions were relatively sparse, few lesions will have resulted from infection by more than one spore; the rate at which this occurred was estimable from the genotype data. Therefore, DNA was extracted directly from the lesion on the leaf disc. Two $4 \mathrm{~mm}$ ball bearings were added to the leaf disc in the microtube and disrupted in an MM2 oscillating mill (Retsch). DNA was then extracted using a DNeasy Plant Mini Kit (Qiagen) following the manufacturer's instructions with all optional steps. DNA was quantified and qualitychecked using a Nanodrop 1000 spectrophotometer (Thermo Scientific) and stored at $-20^{\circ} \mathrm{C}$.

The SSR primers used (Table 1), PCR and thermal cycle conditions, as well as the procedure for genotyping were all carried out as set out in Passey et al. (2016). PCR was repeated on any samples with no product for an SSR marker, alongside a positive control(s), so as to score a null allele, rather than a failed PCR, for that primer pair.

\section{Statistical analysis}

\section{Molecular data}

Allele frequencies were estimated using Powermarker software (Liu \& Muse, 2005). Analysis was run with and without rare alleles (frequency $\leq 0.01$; i.e. an allele appearing only 
once in the orchard in any given year) as very rare alleles have little effect on genetic diversity (Hale et al., 2012). If two alleles were present at a locus it was assumed that the lesion had resulted from infection by more than one spore. If a sample had only one locus with two alleles one was randomly selected. If a sample had multiple loci with more than one allele then the sample was discarded.

We assessed differentiation between populations on the potted trees and the orchard trees by AMOVA (Analysis of Molecular Variance) in Arlequin version 3.5 (Excoffier \& Lischer, 2010). AMOVA significance tests, based on 1023 permutations, were carried out for 'among tree type (Orchard vs. Potted)' and 'among cultivars (Cox vs. Gala)'.

Multi-locus Linkage Disequilibrium (LD) was estimated for scab populations on each tree type for each cultivar to determine whether associations between alleles were compatible with sexual reproduction. LD was calculated by a permutation test (1000 permutations) with Powermarker software. The null hypothesis of the test is that scab from a particular group is in linkage equilibrium, i.e. that the genotype frequency is equal to the product of the allele frequencies (Liu \& Muse, 2005).

\section{Lesions on leaves}

AGGREGATION OF LESIONS. The density of lesions is expected to be higher on leaves of orchard trees than on potted trees because of additional overwintered conidia in the orchard trees. For the same reason, lesions are expected to be more aggregated within an individual leaf on orchard trees than potted trees. We assessed aggregation by fitting the distribution of lesion counts on leaves to a Poisson or negative binomial distribution, separately for potted or orchard trees . We used generalised linear modelling (GLM) to make the fits. In the GLM analysis, cultivar and year were treated as factors; their interaction was not included. Errors were assumed to follow either a Poisson or a negative binomial distribution. Then we used 
the average residual deviance per degree of freedom to compare the goodness of fit of the two models. The best fitting distribution was used in subsequent work,

LESION DENSITY. We tested whether mean lesion counts per leaf were significantly greater for the orchard than for the potted trees using a hurdle model. A limitation of standard count models is that the zeros and the non-zeros (positives) are assumed to come from the same data-generating process; often this type of model cannot account for an excess of zero counts in the data. To overcome this shortcoming, two types of models have been proposed: hurdle models and zero-inflated models (Cameron \& Trivedi, 1998, 2005). For hurdle models, a Bernoulli probability governs the binary outcome of whether a count variate has a zero or positive realisation, similar to the common logistic modelling in GLM. If the realisation is positive (i.e., the hurdle is crossed), positive count data are assumed to be governed by a truncated-at-zero count data model (e.g., Poisson or negative binomial model). On the other hand, zero-inflated models assume that the response variable is a mixture of a Bernoulli distribution and a discrete data-generating process (e.g. Poisson) distribution. Therefore, zero counts can result from a discrete data generating process as well as a Bernoulli process for the zero-inflated models but only from a Bernoulli process for hurdle models.

We chose to use the hurdle models because they enable easy interpretation of differences between potted and orchard trees in the incidence of scabbed leaves and in average lesion counts per scabbed leaf. The incidence of scabbed leaves was modelled as a binomial process and lesion density per scabbed leaf as or a negative binomial process. When fitting hurdle models, the origin of leaves (potted or orchard trees) was used as a factor in both parts of the hurdle model: incidence (logistic model) and density (truncated positive counts model). In addition to the comparison between the potted and orchard trees, year, cultivar and locations in the orchard were included the analysis - but all represented by a single factor of tree pairs: six locations (pairs of trees) within the Cox or Gala section within each year [giving 36 levels 
for the factor 'tree pairs']. Therefore the effects of years and cultivars were already accounted for by the 'tree pairs' factor. We did not include cultivar or year explicitly in the analysis because the purpose of the present study was to study the overall difference in scab development between the potted and orchard trees. GLM was carried out using the MASS package (Venables \& Ripley, 2002) and hurdle models using the pscl package (Zeileis et al., 2008) in $\mathrm{R}$ (version 3.2).

\section{Number of scabbed leaves per shoot}

The variance in the number of infected leaves on a shoot would be expected to be greater in orchard trees due to additional conidial infection localised on particular shoots. For each tree, we have 12-15 shoots. We cannot directly compare variances between trees for two reasons. First, shoots have an unequal number of leaves. Second, the variance of the distribution depends on the mean by the nature of binomial distribution. Therefore, a permutation test, conditioned on the total number of scabbed leaves in a tree, was used to compare the number of infected leaves in each shoot with that expected under the assumption of a random distribution of infected leaves. For each tree, we first conducted the following analysis: (1) find the total number of scabbed leaves; (2) for trees with more than one infected leaf, randomly assigning the same number of infected leaves to the shoots [taking into account the number of leaves on each shoot], (3) calculating the variance among shoots on each tree in the number of scabbed leaves on a shoot, (4) repeating steps 1-3 999 times, (5) calculating the variance of the observed data [we have 1000 variance values for each tree now: 999 variances for simulated data sets and one for the observed], (6) calculating the rank of the observed variance in the 1000 values [if there were ties, using the average rank; rank was calculated in descending order, i.e. the largest value has a rank of 1], and (7) calculating the ratio of the observed variance to the mean of the 999 permutated values. Thus, for each tree the analysis resulted in two values: the rank (frequency with whichthe observed variance would be seen if 
the pattern were random), and the relative size of the observed variance to the mean of a random pattern. Then, ANOVA was applied to assess whether the rank (ln-transformed) or the ratio of variances differed significantly between potted and orchard trees. For the same reasons as outlined above, only tree pairs and the type of tree were included as factors in ANOVA of permutated data. Permutation and ANOVA were implemented in R (version 3.2).

\section{Results}

\section{Molecular data}

In total we screened 396 sampled leaf discs over the three years (2012-2014, Table 2): 202 and 194 samples from potted and orchard trees, respectively. Populations with less than 36 analysed samples were due to: a lack of scab (two potted Cox trees in 2013); samples failing to amplify; or, removal of samples from analysis because they had multiple alleles at more than one locus. A change of capillary in the ABI 3130xl, after the 2012 samples were analysed, led to a $+2 \mathrm{bp}$ shift in markers Vica9/X, Vitc1/82 and Vitg9/129. This was ascertained by running a subset of the 2012 samples and crosschecking against their original allele sizes; an appropriate correction was made to the data. Tests were run with and without rare alleles (frequency $\leq 0.01$ ) of the orchard population in a given year; however, there was no difference in results. Null alleles occur when a mutation in the flanking region of the sequence repeat stops the annealing of the primer and therefore stops amplification during PCR. Statistical tests were run twice, including the null as an extra allele for that marker or excluding the isolate. There were no differences that affected inferences.

AMOVA showed no evidence of difference between the orchard trees and the potted trees in any of the three years, nor any difference between the cultivars $(P>0.3)$.

In 2012 and 2014 all of the multi-locus LD tests showed that the populations were in linkage equilibrium, indicating random mating (Table 3). In 2013 the $V$. inaequalis populations on 
235

Gala potted trees were in linkage equilibrium but the scab populations on the Cox potted and orchard and the Gala orchard trees were in LD (Table 3).

\section{Analysis of scab lesion distributions}

GENERAL RESULTS. Scab was much more severe on Gala than on Cox (Table 4; $\mathrm{P}<$ $0.001)$ : incidence of $5.7 \%( \pm 0.003)(\mathrm{Cox})$ vs $21.6 \%( \pm 0.006)$ (Gala) and average lesion counts per leaf of $0.16( \pm 0.019)($ Cox $)$ vs $1.11( \pm 0.067)$ (Gala). Scab development was more severe in 2012 and 2014 than in 2013 (Table 4). More scab was observed on orchard trees than on potted trees in 2012; however, slightly more scab was seen on potted trees in 2014 than on orchard trees (Table 4). There were only slight differences in the overall scab incidence and density between potted and orchard trees in 2013 (Table 4). Average number of lesions on the scabbed leaves was $4.61( \pm 0.224)$. Overall, there was a larger proportion of leaves with high scab counts on orchard trees than on potted trees, except for Gala in 2014 (Fig. 1).

LESION DISTRIBUTION. A Poisson distribution fitted the count data on potted trees fitted reasonably well (average residual deviance 1.58) but not the for the orchard trees (average residual deviance 3.23). The lack of fit of a Poisson distribution can be seen in Fig. 1, particularly for the susceptible cv. Gala. Both sets of lesion data were equally well described by a negative binomial distribution: average residual deviances were 0.327 and 0.363 for the potted and orchard trees, respectively.

The aggregation of lesions on leaves was further confirmed on fitting the hurdle distributions. A hurdle model based on the negative binomial distribution fitted the data much better than the corresponding model with a Poisson distribution. The hurdle model with a negative binomial error distribution was therefore adopted for further analysis. 
LESION DENSITY. GLM analysis (using hurdle distributions) showed that the incidence of leaves with scab was significantly $(\mathrm{P}<0.001)$ greater on the orchard trees than on the potted trees. For the negative binomial part of the model, the parameter estimate for potted trees was $0.206( \pm 0.063)$ less than that of orchard trees; that is, the odds ratio of being scabbed for potted trees was c. $80 \%$ of corresponding orchard trees. Furthermore, average lesion counts on infected leaves were greater $(\mathrm{P}<0.001)$ on the orchard trees than on the potted trees. Potted trees had an intercept $0.701( \pm 0.140)$ less than that of orchard trees; that is, the average lesion number on potted trees was about $50 \%$ of that on the corresponding orchard trees.

\section{Number of infected leaves per shoot}

The variance in the number of infected leaves on a shoot (expressed as ratio of the observed to the mean of the permuted values) and the rank in a list of random permutations of the observations both differed greatly between potted and orchard trees (Fig. 2). For both variance ratio and log-transform rank variables, residual plots did not suggest any apparent violations of ANOVA assumptions. For potted trees, the ratio of the observed variance in the number of infected leaves on a shoot within each tree to the mean of the permuted values was 0.98 , close to the expected value of 1.0 . For the orchard trees, this ratio was much greater at $1.63\left(\mathrm{~F}_{1,39}=27.2, \mathrm{P}<0.001\right)$. The rank of the observed variance in a permuted dataset (Fig. 2) was much greater in orchard trees $(792)$ than in potted trees $(467)\left(F_{1,39}=25.1, P<0.001\right.$; the average rank of variance of the permuted datasets was necessarily 500).

\section{Discussion}

Previous molecular comparisons of isolates from different cultivars within the same orchard indicated that conidia may overwinter in bud and/or wood scab and act, in addition to ascospores, as a source of primary inoculum (Xu et al., 2013). Several other studies have also 
suggested overwintered conidia are a source of primary inoculum (Becker et al., 1992; Holb et al., 2004, 2005; Gao et al., 2009). In this study we showed that scab lesions on orchard trees were more aggregated on leaves and shoots than on adjacent potted trees not previously exposed to scab (i.e. not exposed to overwintered conidia). Both scab incidence and count data suggest that conidial primary inoculum may have contributed approximately $20-50 \%$ of the total inoculum: incidence was estimated to be reduced by $20 \%$ on potted trees, and lesion number by $50 \%$, averaged over the three years of the study. This interpretation is under the assumptions that infection efficiency by both conidia and ascospores on orchard and potted trees is the same and that both potted and orchard trees are equally susceptible to infection. Infection efficiency in the spring temperatures that the orchard experienced was similar for ascospores and conidia (Reviewed by MacHardy, 1996). The initial infection process should have been completed when the potted trees were returned to the polytunnel; subsequent temperature should not have affected the number of lesions, since we allowed sufficient time for all infections to become visible, predicted on the basis of the relationship of incubation time to temperature (MacHardy, 1996). The likely causes of difference in susceptibility are "softer" tissue in potted plants and lack of resistance priming and induced resistance from phylloplane organisms in the potted plants. Both would produce effects in the opposite direction to those observed.

The scab populations on potted and orchard trees were in linkage equilibrium in both 2012 and 2014. This fits the hypotheses of either predominantly ascospore primary inoculum or no deviation from linkage equilibrium within the conidial primary inoculum, due presumably to no selective changes (detectable with the set of SSRs used) in the population the previous year. In 2013 the population of $V$. inaequalis on potted trees of Gala was in linkage equilibrium but the orchard trees were in linkage disequilibrium. This would be expected if conidia were an important part of the primary inoculum, as the scab on the potted trees would 
be from sexually produced ascospores and therefore from independent sampling, whereas the scab on the orchard trees would be from both (freely recombinant) ascospores and clonal conidia. However, the populations of $V$. inaequalis on both potted and orchard trees of Cox were in linkage disequilibrium in 2013; the potted trees more significantly than the orchard trees. This suggests that unexplained factors influenced our estimates of linkage disequilibrium, so no secure inferences can be drawn. . Although wood scab in heavily infected orchards is commonly observed, it is believed that very few of these wood scab lesions produce viable conidia in spring, indicating that asexually overwintering scab is most likely to result from overwintering in buds (Becker et al., 1992). Although the present study was conducted in an unsprayed orchard (WM132), scab was not very severe and there was no evidence of wood scab present. Furthermore, commercial pruning was applied to the orchard; heavily infected shoots will be likely to have been removed. Thus, conidia that overwintered in the buds are probably the main source of overwintered conidium inoculum in the spring.

We may conclude that ascospores are still the main source of primary inoculum (c. $80 \%$ in this specific orchard) in the spring for temperate growing regions such as Southeast England. Therefore, the current management practice of eliminating leaf debris in late autumn (MacHardy, 1996) needs to be retained. However conidia as primary inoculum cannot be ignored. The relative importance of conidia and ascospores as primary inoculum is likely to vary between orchards and years. In this study we have not compared the differences between years, cultivar or position within an orchard as the aim was to assess the overall importance of conidia primary inoculum. There are many other factors that could affect the relative proportion of conidia as primary inoculum, including pruning, leaf degradation, inseason control efficacy, cultivar, and epidemic severity. Most of the studies suggesting the importance of conidia as part of primary inoculum have been in areas with wet and mild 
winters such as the United Kingdom (Present study; Cook, 1974; Hill, 1975), the Netherlands (Holb et al., 2004, 2005) and west Norway (Stensvand et al., 1996). Conditions in these regions are likely to be both more conducive to faster decomposition of leaf material, reducing ascospore levels, and more likely to allow survival of conidia or mycelia in buds than regions with colder winters. Warmer growing regions, where there is no winter chill necessary for pseudothecia development, only have clonal lineages of the apple scab pathogen (Boehm et al., 2003).

Reducing the amount of inoculum in early season is paramount to good scab control. The main focus of forecast programmes designed to aid effective application of chemical control in spring is currently ascospore release. However, even with a perfect elimination of leaf debris, scab control in the early season is still essential as, based on this work, overwintered conidia are likely to be a source of primary inoculum. Consideration of release of conidia from bud scale should be incorporated into spray guidance programmes. Further, it might also be useful to spray when buds are forming, similar to a strategy being evaluated for reducing overwintering of powdery mildew in apple buds at East Malling.

In summary, we have shown that conidia play an important role as part of the primary inoculum of apple scab in the orchard studied; however, ascospores are still the predominant source. Due to the many factors that can affect the amount of overwintering conidia in orchards, the overall contribution of conidia as primary inoculum is expected to vary considerably with orchards and seasons. Sanitation practices are imperative, for example good winter pruning and removal of leaf litter are both important. Early season sprays are necessary for successful control of scab whether the primary inoculum is from ascospores or overwintered conidia; however traditional spray programmes may have to be revisited in light of these findings. 


\section{Acknowledgements}

362

363

364

365

366

367

368

369

370

371

372

373

374

375

376

377

378

379

380

381

382

383

384

385

386

387

388

389

390

391

392

393

394

395

396

397

398

399

400

401

This research was funded by the Department for Environment, Food and Rural Affairs

(DEFRA).

\section{References}

Becker CM, Burr TJ, Smith CA, 1992. Overwintering of conidia of Venturia inaequalis in apple buds in New York orchards. Plant Disease 76, 121-126.

Beresford RM, Manktelow DWL, 1994. Economics of reducing fungicide use by weather based-disease forecasts for control of Venturia inaequalis in apples. New Zealand Journal of Crop and Horticultural Science 22, 113-120.

Berrie A, Xu X-M, 2003. Managing apple scab (Venturia inaequalis) and powdery mildew (Podosphaera leucotricha) using Adem ${ }^{\mathrm{TM}}$. International Journal of Pest Management 49, 243-249.

Boehm E, Freeman S, Shabi E, Michailides T, 2003. Microsatellite primers indicate the presence of asexual populations of Venturia inaequalis in coastal Israeli apple orchards. Phytoparasitica 31, 236-251.

Cameron AC, Trivedi PK, 1998. Regression Analysis of Count Data. Cambridge: Cambridge University Press.

Cameron AC, Trivedi PK, 2005. Microeconometrics: Methods and Applications. Cambridge: Cambridge University Press.

Cook RTA, 1974. Pustules on wood as sources of inoculum in apple scab and their response to chemical treatments. Annals of Applied Biology 77, 1-9.

Excoffier L, Lischer HEL, 2010. Arlequin suite ver 3.5: A new series of programs to perform population genetics analyses under Linux and Windows. Molecular Ecology Resources 10, 564-567.

Gadoury DM, MacHardy WE, 1986. Forecasting ascospore dose of Venturia inaequalis in commercial apple orchards. Phytopathology 76, 112-118.

Gao L, Berrie A, Yang J, Xu X, 2009. Within- and between-orchard variability in the sensitivity of Venturia inaequalis to myclobutanil, a DMI fungicide, in the UK. Pest management science 65, 1241-9.

Gomez C, Brun L, Chauffour D, Vallée DD Le, 2007. Effect of leaf litter management on scab development in an organic apple orchard. Agriculture, Ecosystems \& Environment 118, 249-255.

Guérin F, Franck P, Loiseau A, Devaux M, Le Cam B, 2004. Isolation of 21 new polymorphic microsatellite loci in the phytopathogenic fungus Venturia inaequalis. Molecular Ecology Notes 4, 268-270.

Hale ML, Burg TM, Steeves TE, 2012. Sampling for microsatellite-based population genetic studies: 25 to 30 individuals per population is enough to accurately estimate allele frequencies. PLOS ONE 7, e45170. 
Hill SA, 1975. The importance of wood scab caused by Venturia inaequalis (Cke.) Wint. as a source of infection for apple leaves in the spring. Phytopathologische Zeitschrift 82, 216-223.

Holb I, Heijne B, Jeger M, 2004. Overwintering of conidia of Venturia inaequalis and the contribution to early epidemics of apple scab. Plant Disease 88, 751-757.

Holb IJ, Heijne B, Jeger MJ, 2005. The widespread occurrence of overwintered conidial inoculum of Venturia inaequalis on shoots and buds in organic and integrated apple orchards across the Netherlands. European Journal of Plant Pathology 111, 157-168.

Liu K, Muse S V, 2005. PowerMarker: an integrated analysis environment for genetic marker analysis. Bioinformatics 21, 2128-2129.

MacHardy WE, 1996. Apple Scab: Biology, Epidemiology, and Management. St Paul, MN: American Phytopathological Society.

Passey TAJ, Shaw MW, Xu X-M, 2016. Differentiation in populations of the apple scab fungus Venturia inaequalis on cultivars in a mixed orchard remain over time. Plant Pathology.

Stensvand A, Amundsen T, Semb L, 1996. Observations on wood scab caused by Venturia inaequalis and V. pirina in apple and pear in Norway. Norwegian Journal of Agricultural Sciences 10, 533-540.

Sutton DK, MacHardy WE, Lord WG, 2000. Effects of shredding or treating apple leaf litter with urea on ascospore d ose of Venturia inaequalis and disease buildup. Plant Disease 84, 1319-1326.

Venables WN, Ripley BD, 2002. Modern Applied Statistics with S, 4th edition. New York: Springer.

Vincent C, Rancourt B, Carisse O, 2004. Apple leaf shredding as a non-chemical tool to manage apple scab and spotted tentiform leafminer. Agriculture, Ecosystems \& Environment 104, 595-604.

Xu X, Harvey N, Roberts A, Barbara D, 2013. Population variation of apple scab (Venturia inaequalis) within mixed orchards in the UK. European Journal of Plant Pathology 135, 97-104.

Xu X, Roberts T, Barbara D, Harvey NG, Gao L, Sargent DJ, 2009. A genetic linkage map of Venturia inaequalis, the causal agent of apple scab. BMC Research Notes 2, 163.

Zeileis A, Kleiber C, Jackman S, 2008. Regression models for count data in R. Journal of Statistical Software 27, 1-25. 


\begin{tabular}{llll}
\hline \multicolumn{2}{l}{ Table 1 Sequences (5'-3') for SSR primer pairs used to genotype apple scab isolates } \\
\hline SSR & Fluorescent label-Forward primer & Reverse Primer & $\begin{array}{l}\text { Allele } \\
\text { range }\end{array}$ \\
\hline EMVi029 $^{\text {a }}$ & HEX-ACGAGTCCAGGTCTCACAG & TGTTGACGGTCACGGTGTAT & $170-252$ \\
Vica9/X $^{\text {b }}$ & FAM-TCGCGCATCACTATCTACAC & AGACAGGAATGTGGTGGAAG & $219-247$ \\
Vica10/154 & HEX-CCTCCTCCTATTACTCTCG & CTGAAGCGAACCTATGTCC & $100-168$ \\
Vicacg8/42 & FAM-TGTCAGCCACGCTAGAAG & CACCGGACGAATCATGC & $200-240$ \\
Vict1/130 $^{\text {b }}$ & FAM-GATTGGTGACGCATGTGT & GCTGGAGATTGCGTAGAC & $148-164$ \\
Vitc1/82 & HEX-ACTGTCTCTAGGCGAAAG & ACTTGGAAGCTCGCTAAG & $227-243$ \\
Vitc2/16 & FAM-ACATTGACGAAGACGAGC & TACAATTGAGGCGTGTCC & $153-169$ \\
Vitg9/129 & FAM-CTAATTCAACTCGCTGCGTC & TTTCAGCCAGCTAACCTAGG & $277-291$ \\
\hline
\end{tabular}

${ }^{\mathrm{a} X u}$ et al., 2009

${ }^{\mathrm{b}}$ Guérin et al., 2004

439

Table 2 Number of leaf discs with scab lesions screened for SSR markers to compare populations from potted trap trees and orchard trees

\begin{tabular}{lllllll}
\hline Type & \multicolumn{2}{c}{2012} & \multicolumn{3}{c}{2013} & \multicolumn{2}{c}{2014} \\
\cline { 2 - 7 } & Cox & Gala & Cox & Gala & Cox & Gala \\
Potted & 36 & 36 & 25 & 35 & 35 & 35 \\
Orchard & 31 & 29 & 34 & 31 & 36 & 33 \\
\hline
\end{tabular}

Table 3 Significance results in test for Linkage Disequilibrium of $V$. inaequalis populations of potted and orchard trees in different cultivars in an orchard in Southeast England

\begin{tabular}{lllll}
\hline \multirow{3}{*}{ Cox } & Population(s) & 2012 & 2013 & 2014 \\
\cline { 2 - 5 } Gala & Orchard & 1.00 & 0.01 & 1.00 \\
& Potted & 1.00 & $<0.001$ & 1.00 \\
& Orchard & 1.00 & 0.01 & 1.00 \\
& Potted & 1.00 & 1.00 & 1.00 \\
\hline
\end{tabular}

449

Table 4 Incidence of leaves with scab and average number of lesions per leaf on orchard and potted trees of cvs. Cox and Gala in an orchard in Southeast

England

\begin{tabular}{|c|c|c|c|c|c|c|}
\hline \multirow{3}{*}{ Type } & 2012 & & 2013 & & 2014 & \\
\hline & Cox & Gala & Cox & Gala & Cox & Gala \\
\hline & \multicolumn{6}{|c|}{ Number of leaves assessed } \\
\hline Potted & 1201 & 1105 & 738 & 687 & 1051 & 602 \\
\hline \multirow[t]{2}{*}{ Orchard } & 917 & 850 & 830 & 951 & 797 & 686 \\
\hline & \multicolumn{6}{|c|}{ Incidence of leaves with scab } \\
\hline Pot & 0.06 & 0.171 & 0.049 & 0.180 & 0.049 & 0.261 \\
\hline \multirow[t]{2}{*}{ Orchard } & 0.108 & 0.301 & 0.047 & 0.181 & 0.017 & 0.230 \\
\hline & \multicolumn{6}{|c|}{ Average lesion counts } \\
\hline ot & 0.118 & 0.536 & 0.172 & 0.646 & 0.059 & 1.228 \\
\hline Orchard & 0.358 & 2.414 & 0.263 & 1.077 & 0.025 & 0.828 \\
\hline
\end{tabular}



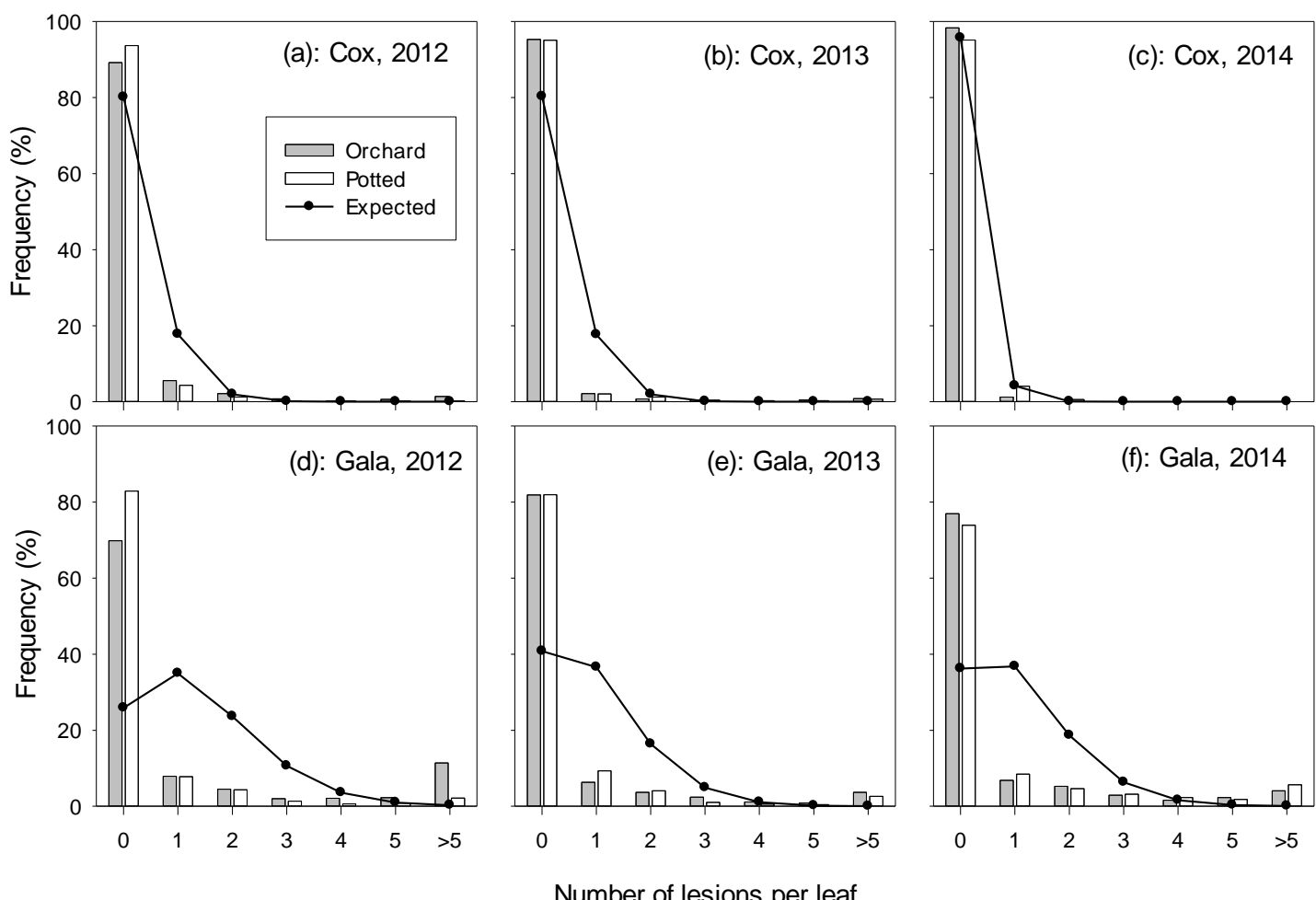

Number of lesions per leaf

Figure 1. Distribution of apple scab lesions on individual leaves collected from potted trees (non-shaded bar) and corresponding orchard trees (shaded bar) of two cultivars in three years when both types of trees were exposed to the same conditions at the same locations. In addition the expected frequency assuming a Poisson (random) distribution for number of lesions on individual leaves is also shown (line). Observed data has a higher frequency than expected for leaves with no lesions and more than four lesions per leaf indicating aggregation of lesions within a single leaf.
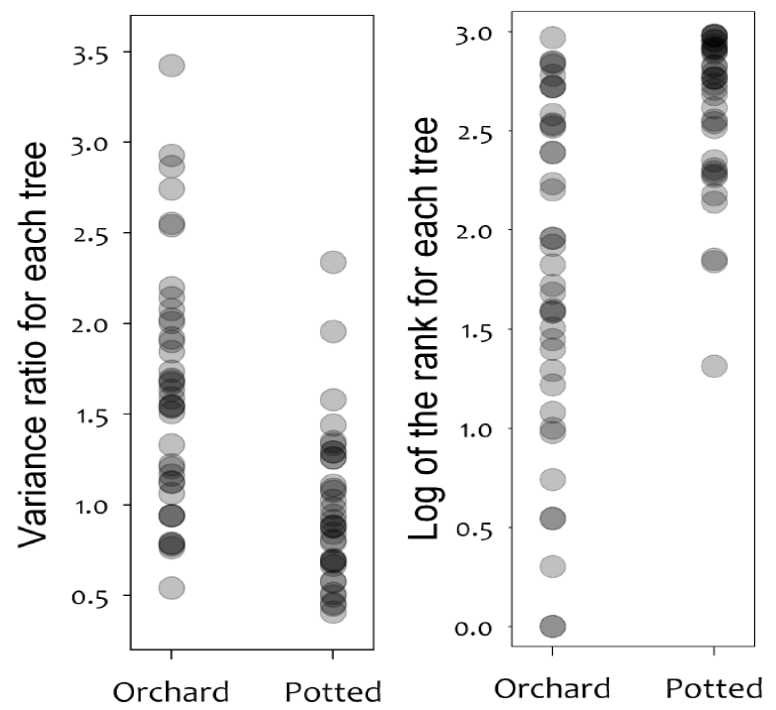

Figure 2. Plot of the ratio between the observed variance in the number of scabbed apple leaves in each shoot within each tree with the average variance of 999 permutations assuming random distribution of infected leaves, and the log of the rank of the observed 
472 variance among the 1000 variance values (999 permutated and one observed; in the 473 descending order - i.e. the largest has the rank of one). Depth of grey indicates overlaying of 474 observations. The rank of observed variance was significantly different $(\mathrm{P}<0.001)$ between 475 orchard and potted trees in this Southeast England orchard. 